\title{
Utility of flow cytometry and gene rearrangement analysis in tissue and blood of patients with suspected cutaneous T-cell lymphoma
}

\author{
JULIE D. GIBBS ${ }^{1 *}$, SOPHIA MA ${ }^{2 *}$, ANNA KIM $^{3}$, LUCIA SEMINARIO-VIDAL $^{3}$, LUBOMIR SOKOL $^{4}$, \\ HAILING ZHANG ${ }^{5}$, XIAOHUI ZHANG ${ }^{5}$, ELIZABETH SAGATYS ${ }^{5}$, PEI-LING CHEN ${ }^{5}$ and JANE L. MESSINA ${ }^{6}$ \\ ${ }^{1}$ Bay Pines VA Medical Center, St. Petersburg, FL 33744; ${ }^{2}$ Sutter Health, San Ramon, CA 94583; \\ ${ }^{3}$ Department of Dermatology and Cutaneous Surgery, University of South Florida Morsani College of Medicine; \\ Departments of ${ }^{4}$ Malignant Hematology and ${ }^{5}$ Hematopathology and Laboratory Medicine, \\ ${ }^{6}$ Anatomic Pathology, H. Lee Moffitt Cancer Center and Research Institute, Tampa, FL 33612, USA
}

Received April 16, 2020; Accepted October 30, 2020

DOI: 10.3892/or.2020.7865

\begin{abstract}
Cutaneous T-cell lymphoma (CTCL) is difficult to diagnose at an early stage. Current diagnostic tools include clinical examination, histomorphologic analysis, immunohistochemistry, flow cytometry of peripheral blood and/or lesional tissue, and evaluation of T-cell receptor (TCR) clonality by gene rearrangement analysis (TCRGR). Advances in genomic sequencing, including high-throughput sequencing (HTS), can be used to identify specific clones of rearranged TCR genes. Even with all of these tools, CTCL can take as long as a decade to definitively diagnose, potentially delaying treatment options and causing patient anxiety. This study aimed to evaluate the performance of the various ancillary testing modalities used to diagnose early-stage CTCL. In a subset of patients the performance of HTS was compared to flow cytometry and conventional TCRGR analysis via polymerase chain reaction (PCR). Fifty-five patients, with a total of 68 skin biopsies and peripheral blood draws, were evaluated using flow cytometry, PCR-TCRGR, and HTS-TCRGR to determine the sensitivity and specificity of each ancillary test. In tissue biopsies, flow cytometry (64\%), PCR (71\%) and HTS (69\%) shared similar sensitivities; flow cytometry had the highest specificity (93\%), followed by HTS (86\%) and PCR (76.9\%). However, flow cytometry and PCR had insufficient DNA quantities in 29 and $15 \%$ of the specimens, respectively. Peripheral blood testing was less sensitive than tissue testing (flow cytometry $14 \%$, PCR $41 \%$, HTS 33\%); in peripheral blood, HTS was the most specific test (flow cytometry, $70 \%$; PCR, $62.5 \%$; and
\end{abstract}

Correspondence to: Dr Jane L. Messina, Department of Anatomic Pathology, H. Lee Moffitt Cancer Center and Research Institute,12902 Magnolia Drive, Tampa, FL 33612, USA

E-mail: jane.messina@moffitt.org

${ }^{*}$ Contributed equally

Key words: cutaneous T-cell lymphoma, pathologic diagnosis, gene rearrangement analysis, T-cell receptor sequencing, flow cytometry
HTS, $100 \%$ ). HTS is a highly specific molecular test for identifying CTCL in peripheral blood and skin biopsy specimens; however, our findings suggest a need for a continued search for more sensitive tests for early-stage CTCL.

\section{Introduction}

Cutaneous T-cell lymphoma (CTCL) is the most common type of lymphoma involving the skin. It accounts for 80 to $85 \%$ of all primary cutaneous lymphomas, with approximately 1,000 individuals in the US newly diagnosed annually $(1,2)$. There are multiple variants of CTCL, which are classified on the basis of histopathologic and clinical features. The World Health Organization's classical categorization of CTCL is divided into indolent clinical behavior and aggressive behavior, such as that expressed by Sèzary syndrome lymphomas/leukemias (1,3-5). Mycosis fungoides is the most common subtype of CTCL, comprising nearly $50 \%$ of all primary cutaneous lymphomas $(2,3,6)$. There are 4 distinct clinical $(\mathrm{T})$ stages of mycosis fungoides in the skin, which are based on the appearance of patch, plaque, tumor and erythroderma, respectively (7). A complete history and physical examination, including a full skin evaluation, must be performed to identify lesions suspicious for CTCL, and a diagnostic skin biopsy is required to make a definitive diagnosis. Histologic criteria that have proven useful for the diagnosis of mycosis fungoides include epidermotropism, Pautrier microabscesses, dense lymphoid infiltrate, perinuclear halos, and enlarged hyperchromatic cerebriform nuclei (6). However, these histologies are more commonly observed in advanced plaque and tumor stages of CTCL and are subtle or even absent in early patch-stage CTCL (8). Early-stage CTCL demonstrates borderline histologic changes with variable inflammatory reaction patterns (9). Pautrier microabscesses are absent in the majority of patch-stage cases (10).

Accordingly, early-stage CTCL is challenging to diagnose for both the dermatologist and dermatopathologist (11). There is significant overlap between the clinical and histopathologic features of CTCL and many inflammatory skin diseases, especially during the early-patch stage. Clinically, CTCL typically presents as erythematous, scaly macules, patches, 
and plaques that can be nearly indistinguishable from the presentations of eczema, psoriasis, and lichenoid dermatoses. The histology of early-stage CTCL can also show variable spongiotic, psoriasiform, and/or interface patterns of dermatitis. Immunohistochemical (IHC) stains can aid diagnosis by demonstrating predominant $\mathrm{CD} 4$ positivity within the T-cell infiltrate; however, IHC analyses are limited by the significant overlap between the early-stage features of CTCL and chronic dermatitis. A typical IHC panel consists of pan-T-cell markers as well as CD4 and CD8. Findings that support a diagnosis of CTCL include an elevated CD4 to CD8 ratio (12) and/or loss of pan-T-cell markers, especially CD5 or CD7. However, loss of T-cell markers, such as CD2, CD3, CD4, or CD5, by the neoplastic $\mathrm{T}$ cells typically occurs in advanced-stage CTCL but is less common in early-stage lesions. Furthermore, loss of CD7 is not specific for CTCL and has been described in inflammatory conditions (13-16).

Because of these diagnostic challenges, the diagnosis of CTCL is often delayed; the mean time from initial presentation to an accurate diagnosis of CTCL is approximately 4 to 6 years $(17,18)$. Fortunately, skin-limited CTCL is often indolent, and patients with patch/plaque-stage disease have a life expectancy similar to controls matched for age, sex and race (7). Nonetheless, the advent of effective therapies for early-stage disease, as well as the desire to alleviate patient anxiety, has led to the search for more sensitive and specific ancillary methods for an early diagnosis.

These putative ancillary diagnostic techniques are often employed in the initial workup of CTCL. They include flow cytometry of skin biopsy tissue and peripheral blood and molecular evaluation of T-cell clonality using T-cell receptor gene rearrangement by polymerase chain reaction (TCGR-PCR), with PCR being the standard molecular technique for TCRGR analysis. Although PCR is a useful tool, its ability to identify TCRGR can be limited. Especially in early-stage lesions, the identification of a clone using PCR is associated with a high false-negative rate (19). Flow cytometry of peripheral blood is mostly used in the evaluation of erythrodermic patients, but its sensitivity and specificity in early-stage disease has only rarely been investigated. A recent study demonstrated sensitivity of $78 \%$ and specificity of $71 \%$ in a group of 128 samples of various stages, including early-stage mycosis fungoides (20) while an earlier study showed lower sensitivity of only $6 \%$ in 440 cases (21). Likewise, flow cytometry performed on skin biopsies is not in widespread use, although a small study showed promising sensitivity of $75 \%$ (22). Therefore, there remains considerable debate concerning the value of all of these ancillary studies, particularly in regards to early-stage or suspected CTCL.

With the advent of next generation sequencing, the use of high-throughput single cell T-cell receptor sequencing-high throughput screening (HTS) to identify TCRGR has shown better specificity in distinguishing definitive CTCL from inflammatory processes (23). The tumor clone frequency quantified by using HTS may be used to identify patients with early-stage CTCL who are at a higher risk of progression (24). Additionally, HTS has been shown to be more sensitive and specific than flow cytometry in identifying minimal residual disease in patients with Sèzary syndrome (25).
However, the optimal use of T-cell receptor (TCR) clonality and flow cytometry studies, especially for evaluation of the patient with suspected and/or early-stage CTCL, has not been clearly defined. A better understanding of the yield of these ancillary tests can facilitate more cost-effective care. Furthermore, defining the tests with optimal yield may obviate the need for repeated clinical visits and/or additional biopsies to establish a definitive diagnosis. In the current study, we describe the yield of the more commonly used ancillary tests and the more recently available TCR-HTS studies that are performed on skin biopsy tissue and peripheral blood specimens from patients who were evaluated for suspected CTCL at a cutaneous lymphoma referral clinic.

\section{Patients and methods}

Patients. Following approval of the Institutional Review Board of the University of South Florida, we retrospectively reviewed the medical records of patients who were referred to the multidisciplinary cutaneous lymphoma clinic at our tertiary care cancer center between April 1, 2017 and December 6, 2017. We analyzed sequentially all new patients who were referred for skin disease which was suspected clinically to be a new diagnosis of CTCL, as well as patients with a prior history of CTCL who had responded to treatment but were being evaluated for a suspected new clinical recurrence, and who underwent skin biopsy, flow cytometry of skin and/or peripheral blood, and gene rearrangement analysis of skin biopsy as part of their workup. Patients were excluded if they were not clinically suspicious for CTCL before undergoing a diagnostic biopsy or had no identifiable lymphoid infiltrates on pathologic examination. Patients with a diagnosis of CTCL who had biopsies to evaluate for progression of already established CTCL were also excluded.

Demographic data such as age, sex, and race were obtained. The clinical appearance of skin lesions (patch, plaque, tumor) at the time of presentation and the diagnostic results of flow cytometry, TCR, and HTS analyses, along with the final consensus diagnosis of each patient, were recorded. If lesions of $>1$ morphology were present, the clinical categorization was that of the most advanced morphology.

Skin biopsy evaluation. Each patient underwent one skin biopsy, either a 3-mm punch biopsy or a shave biopsy measuring at least $4 \mathrm{~mm}$, of each skin lesion with patch, plaque, and/or tumor morphology. Biopsies were submitted in formalin for hematoxylin and eosin (H\&E) and IHC evaluation, TCR-PCR and/or TCR-HTS analysis. Most patients underwent an additional 3-mm punch biopsy which was submitted fresh for flow cytometry analysis in some cases; the shave or punch for pathologic diagnosis was bisected and one half was submitted fresh for flow cytometry with the remainder for H\&E. H\&E-stained slides were evaluated for histologic features diagnostic of CTCL, including band-like dermal lymphocytes, epidermotropism, Pautrier microabscesses, follicular mucinosis, cytologic atypia of lymphocytes, and wiry papillary dermal fibrosis.

Immunohistochemistry. IHC staining was performed on a Ventana immunostainer (Ventana Benchmark Ultra, Roche Diagnostics). Antibodies included CD2 (Cell Marque); 
CD3, CD4, CD5, CD7, CD20, CD30 (Ventana/Roche); and CD8 (Dako/Aligent). Positive staining was visualized with diaminobenzidine chromogen.

Flow cytometry. Fresh tissue biopsies submitted for flow cytometry analyses were transported at room temperature in Roswell Park Memorial Institute (RPMI)-1640 media to the flow cytometry laboratory and manually cut into 1 to $2 \mathrm{~mm}^{3}$ pieces with a scalpel. They were then loaded on a Medimachine sample preparation system (BD Biosciences) and processed per manufacturer's instructions. Briefly, this system uses disposable polyethylene Medicon chambers with immobile stainless-steel screens that contain microblades around each hole in the screen, which allow for tissue disaggregation. After $45 \mathrm{sec}$, the disaggregated tissue was collected and filtered through a $50-\mu \mathrm{m}$ Filcon filter membrane, then centrifuged for $5 \mathrm{~min}$ at $250 \mathrm{x} \mathrm{g}$. The pellet was resuspended in RPMI-1640 medium. Cell count and viability assessments were performed; the concentration was between $5 \times 10^{5}$ to $1 \times 10^{6}$ cells $/ \mathrm{ml}$. Aliquots of the resulting cell suspension were stained in the dark for $15 \mathrm{~min}$ at room temperature with combinations of monoclonal antibodies conjugated to fluorescein isothiocyanate, phycoerythrin-Texas Red, peridinin chlorophyll protein CyChrome 5.5, phycoerythrin-cyanine 7, allophycocyanin, allophycocyanin-Alexa Fluor 700, allophycocyanin-Alexa Fluor 750, Pacific Blue, and Krome Orange. Red blood cells were lysed with BD FACS lysing solution (BD Biosciences), and nucleated cells were resuspended in phosphate-buffered saline containing $2 \%$ paraformaldehyde. Events were acquired on a Gallios flow cytometer (Beckman Coulter). The one-tube panel for skin consists of CD2, CD26, CD3, CD5, CD7, CD30, CD19, CD4, CD8, and CD45 (Beckman Coulter). Listmode files were analyzed using Kaluza version 1.2 (Beckman Coulter). The lymphoid population was obtained on a dot plot of ungated forward scatter/side scatter and CD45/side scatter gating for all lymphocytes. A positive flow cytometry is diagnosed when a distinct aberrant $\mathrm{T}$-cell cluster is identified, such as a $\mathrm{T}$ cell population with aberrant loss of CD7, CD26, or any other pan-T-cell markers.

Flow cytometry analyses of peripheral blood were conducted as follows. Peripheral blood cell counts were calculated by using an XE-2100 automated hemocytometer (Sysmex, Hyogo, Japan). Aliquots of $100 \mu 1$ of whole blood in sodium heparin were incubated in the dark for $15 \mathrm{~min}$ at room temperature with combinations of monoclonal antibodies conjugated to the above-described fluorochromes. Red blood cells were lysed with BD FACS lysing solution (BD Biosciences), and nucleated cells were resuspended in phosphate-buffered saline containing $2 \%$ paraformaldehyde. Events were acquired on a Gallios flow cytometer (Beckman Coulter). The panel consisted of 5 tubes containing the following combinations: CD2, CD26, CD3, CD5, CD7, CD30, CD19, CD4, CD8, and CD45; TCR $\gamma / \delta$, TCR $\alpha / \beta$, CD3, CD279, CD10, CD194, CD25, CD4, CD8, and CD45; CD45RA, CD52, CD62L, CD56, CD7, CD16, CD4, CD3, CD57, and CD45; TCR $\gamma / \delta$, TCR $\alpha / \beta$, CD8, CD5, CD7, CD16, CD56, HLA-DR, CD57, and CD45; and $\kappa$-light chain, $\lambda$-light chain, CD8, CD5, CD10, CD20, CD19, CD4, CD3, and CD45. All antibodies were obtained from Beckman Coulter. Lymphoid populations were identified and categorized. Fig. 1 provides a summary of the antigens evaluated via flow cytometry analyses of skin biopsies and peripheral blood.

TCRGR by PCR. This test was performed on DNA prepared from paraffin-embedded skin biopsy tissue and/or an aliquot of the cell suspension in RPMI-1640 medium produced by the flow cytometry laboratory. Total cellular DNA was extracted and PCR amplification was performed on 5 multiplex PCR tubes with analyte specific reagent Biomed-2 primers (Invivoscribe Technologies) targeting TCR $\mathrm{V} \beta, \mathrm{D} \beta, \mathrm{J} \beta, \mathrm{V} \gamma$, and $\mathrm{J} \gamma$ regions. The products were separated and detected by capillary gel electrophoresis on the ABI PRISM 3130xl Genetic Analyzer (Applied Biosystems). A positive PCR result is defined as a single peak that is $>2.5 \mathrm{x}$ larger than adjacent polyclonal peaks.

TCRGR by HTS. DNA extracted from paraffin-embedded skin biopsy specimens was submitted for TCR-HTS to Adaptive Biosciences for Clonoseq testing. TCR- $\beta$ complementary determining region 3 (CDR3) was targeted and amplified by using multiplex PCR, with bias-controlled V and $\mathrm{J}$ gene primers. The amplified segments were sequenced using HTS. Cases were considered positive if there was at least one unique sequence comprising $>5 \%$ of all sequences identified. For these cases, the number and sequence(s) of clones containing unique CDR3 regions/1 million cells reported. Cases with unique sequences at lower than $5 \%$ frequency were considered polyclonal.

Data analysis. For each of the testing modalities, the number of adequate specimens per patient tested, as well as the rate of positivity of adequate specimens was compiled for each lesion morphology. The sensitivity (true positives/true positives + false negatives) and specificity (true negative/true negatives + false positives) of TCR-PCR, TCR-HTS, and flow cytometry studies performed were calculated for each patient using the final integrated diagnosis (CTCL or not CTCL), which was based on the integration of clinical, histopathologic, and molecular analyses of all biopsy specimens from that patient. A final integrated diagnosis of mycosis fungoides included at least 2 of the following findings: A chronic cutaneous eruption clinically suspicious for CTCL exhibiting an abnormal clone of CD26-expressing $\mathrm{T}$ cells on flow cytometry analyses, a positive TCRGR for $\beta$ and $\gamma$ genes by PCR and/or HTS, an epidermotropic lymphoid infiltrate on $\mathrm{H} \& \mathrm{E}$ staining with an altered CD4 to CD8 ratio (either $>5$ or $<2$ ), and/or a loss of pan-T-cell antigen(s), such as CD2, CD5, or CD7.

\section{Results}

A total of 55 patients referred to the cutaneous lymphoma clinic between April 1, 2017 and December 6, 2017 met the inclusion criteria. The patients ranged from 21 to 85 years of age. Clinicopathologic characteristics are summarized in Table I. Representative examples of results for a patient with patch-stage disease (clinical images, histology, flow cytometry and PCR results) are shown in Fig. 2, and for plaque disease in Fig. 3. 
Table I. Clinicopathologic characteristics of the cases.

\begin{tabular}{|c|c|c|c|c|}
\hline Characteristics & Patch morphology & Plaque morphology & Tumor morphology & Erythroderma \\
\hline \multicolumn{5}{|l|}{ Age in years } \\
\hline Median & 62 & 58 & 68 & 77 \\
\hline Range & $29-80$ & $21-76$ & $52-69$ & $66-85$ \\
\hline \multicolumn{5}{|l|}{ Sex, n (\%) } \\
\hline Male & $15(68)$ & $13(54)$ & $3(100)$ & $3(50)$ \\
\hline Female & $7(32)$ & $11(46)$ & $0 \quad(0)$ & $3(50)$ \\
\hline \multicolumn{5}{|l|}{ BSA, n (\%) } \\
\hline$<1 \%$ & $3(14)$ & $1 \quad(4)$ & 0 & 0 \\
\hline $1-9 \%$ & $10(45)$ & $12(50)$ & 1 (33) & 0 \\
\hline $10-79 \%$ & $8(36)$ & $5(21)$ & 0 & 0 \\
\hline$>80 \%$ & 0 & 0 & 0 & $6(100)$ \\
\hline Unknown & $1 \quad(5)$ & 7 (29) & $2(67)$ & 0 \\
\hline \multicolumn{5}{|l|}{ Clinical presentation, $\mathrm{n}(\%)$} \\
\hline New patients & $16(73)$ & $22(88)$ & $2(67)$ & $3(50)$ \\
\hline Recurrent patients & $6(27)$ & $3(12)$ & $1 \quad(33)$ & $3(50)$ \\
\hline \multicolumn{5}{|l|}{ Final diagnosis, n (\%) } \\
\hline MF & $14(64)$ & $16(67)$ & $1 \quad(33)$ & $4(67)$ \\
\hline Non-MF & $8(36)$ & $8(33)$ & $2(67)$ & $2(33)$ \\
\hline Patients (total, $\mathrm{N}=55$ ), $\mathrm{n}$ & 22 & 24 & 3 & 6 \\
\hline
\end{tabular}

BSA, body surface area; MF, Mycosis fungoides.
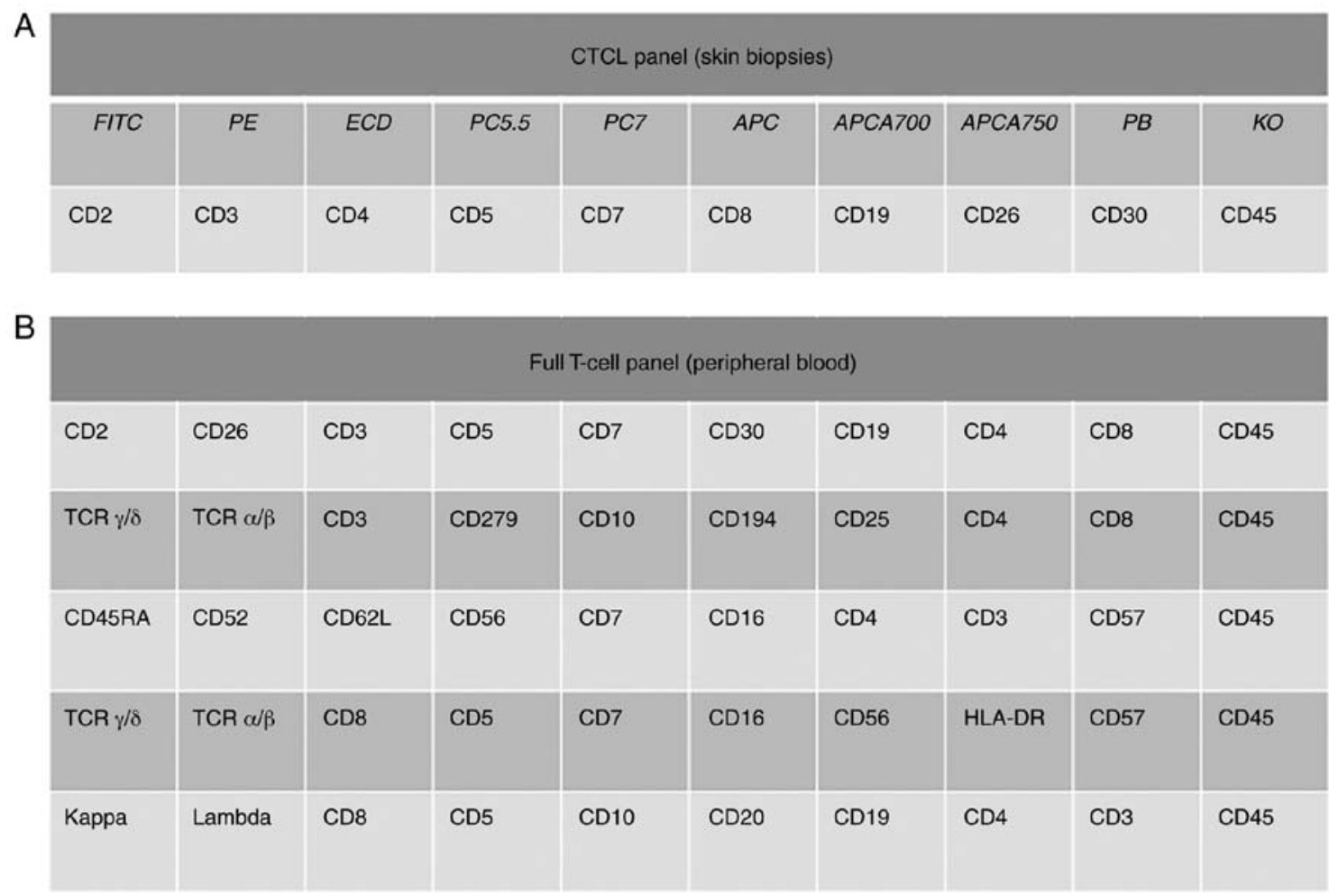

Figure 1. T-cell panel flow cytometry markers analyzed in (A) biopsy tissue and (B) peripheral blood. CTCL, cutaneous T-cell lymphoma.

Clinical lesions that were biopsied included patches ( $\mathrm{n}=45$ biopsies from 28 patients), plaques ( $\mathrm{n}=43$ biopsies from 24 patients), nodules/tumors ( $\mathrm{n}=5$ biopsies from
3 patients), and erythroderma ( $\mathrm{n}=9$ biopsies from 6 patients); 6 of these patients had both patches and plaques submitted. One hundred and two (102) skin biopsy specimens were 

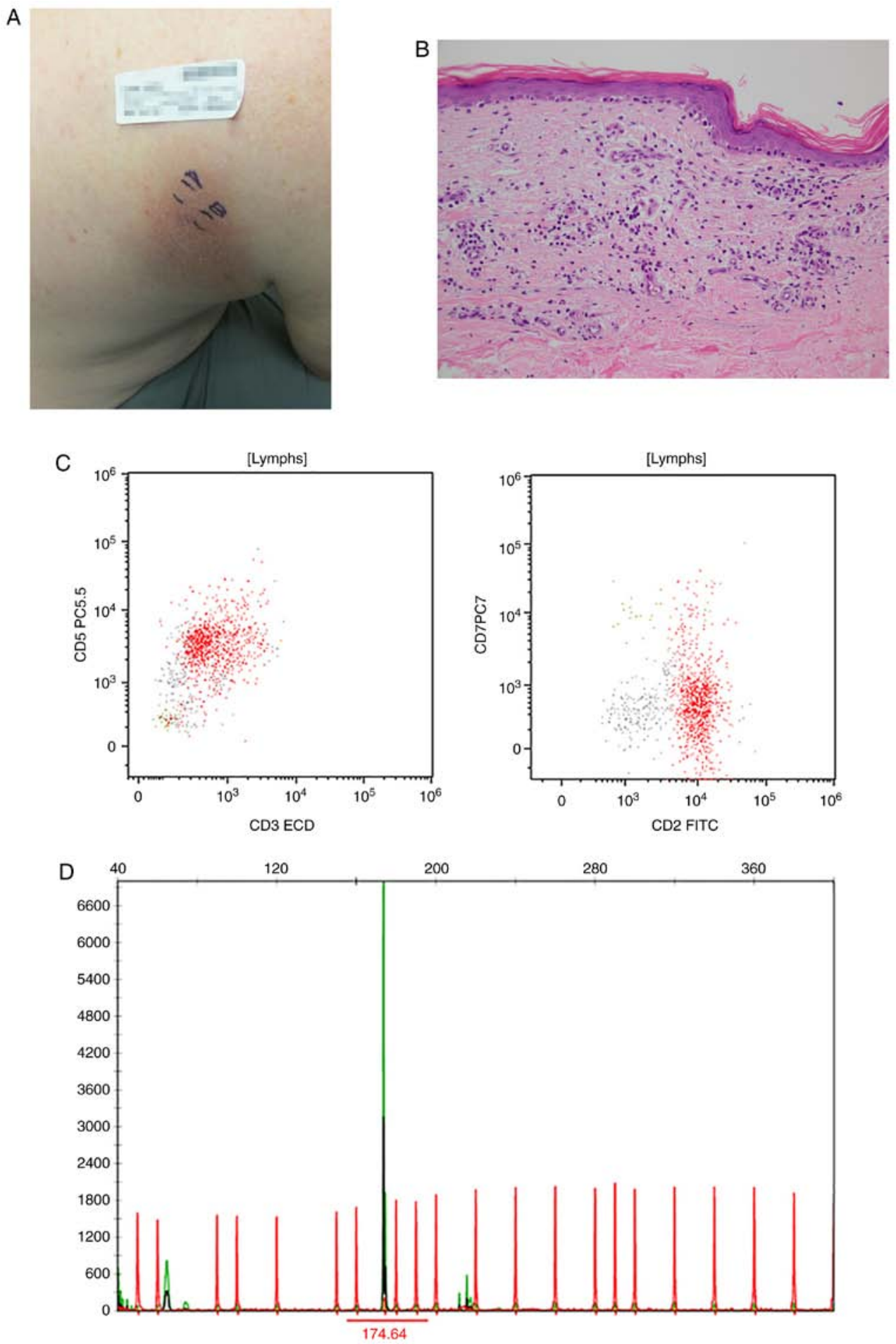

Figure 2. Patient with patch-stage mycosis fungoides showing (A) erythematous scaly patch and (B) sparse dermal infiltrate of hyperchromatic lymphocytes with epidermotropism (magnification, x20). (C) Atypical population of CD2/CD3-positive T lymphocytes expressing dim CD2 and lacking CD7 as identified via flow cytometry from skin tissue. (D) Single prominent peak showing TCR gamma gene clonal rearrangement. TCR, T-cell receptor.

submitted for H\&E histologic evaluation, with associated flow cytometry ( $\mathrm{n}=59$ tests from 53 patients), TCR-PCR $(\mathrm{n}=50$ tests from 50 patients), and/or TCR-HTS ( $\mathrm{n}=26$ tests from 23 patients) studies performed. There were 21 concurrent tissue TCR-PCR and TCR-HTS specimens. Peripheral blood specimens were submitted for flow cytometry (51 from 51 patients), TCR-PCR (50 from 50 patients) and TCR-HTS (16 from 16 patients), with 15 concurrent TCR-PCR and TCR-HTS blood specimens. Thirty-five (64) patients had a final integrated diagnosis of CTCL. Representative clinical images, H\&E slides, flow cytometry, and TCRGR results are presented for a patient with patch-stage mycosis fungoides (Fig. 3) and plaque stage mycosis fungoides. A representative example of immunohistochemical staining used to support a diagnosis of plaque-stage mycosis fungoides is shown in Fig. S1. An example of a high-throughput sequencing report on a patient with plaque-stage mycosis fungoides is shown in Fig. S2. The diagnoses of the remaining 20 patients 
A
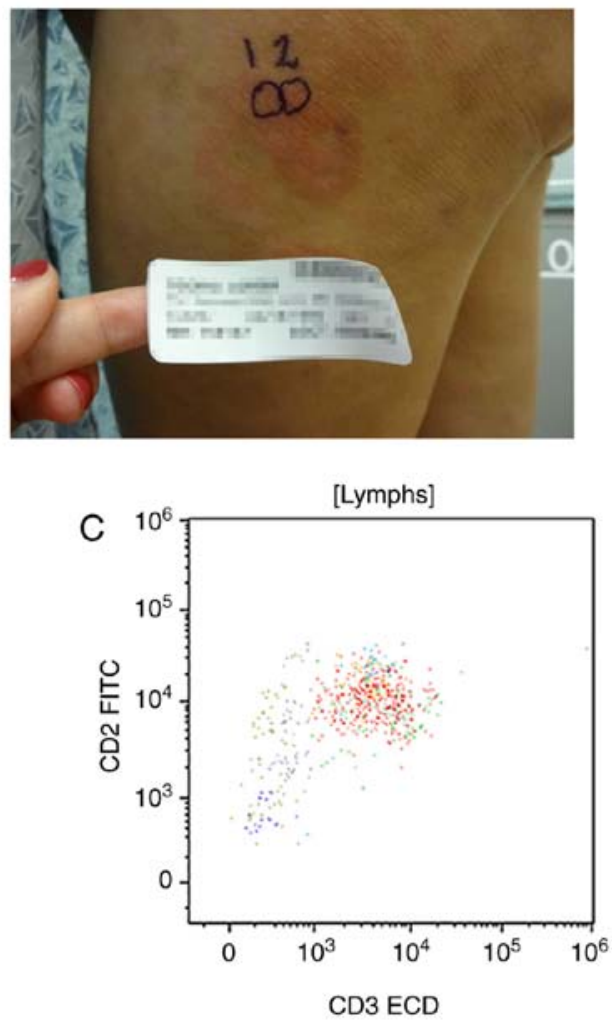

B
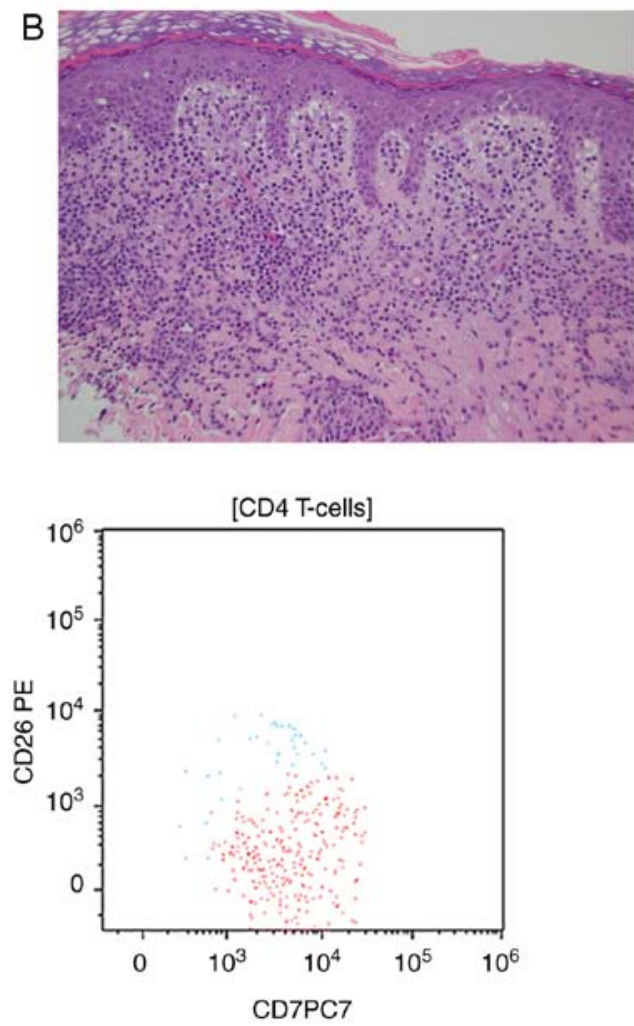

D
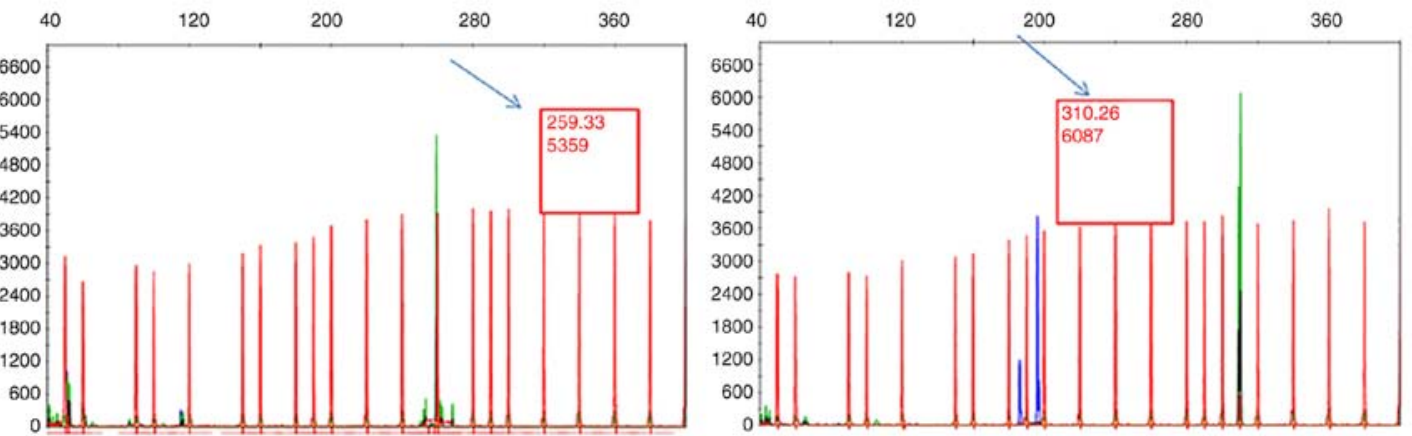

Figure 3. Patient with plaque-stage mycosis fungoides, showing (A) erythematous scaly vaguely annular plaque and (B) dense, band-like dermal infiltrate of hyperchromatic cerebriform lymphocytes with epidermotropism (magnification, $\mathrm{x} 20$ ). (C) Population of T lymphocytes with expression of CD2 and CD3 but aberrant loss of aberant loss of CD7 and CD26 as identified via flow cytometry from skin tissue. (D) Monoclonal peaks identified via beta and gamma gene rearrangement analyses.

were pseudolymphoma $(n=7)$, no evidence of/inactive disease $(n=4)$, atypical lymphoid infiltrate not diagnostic for CTCL $(n=1)$, lymphomatoid papulosis $(n=1)$, superficial perivascular dermatitis $(n=2)$, phototoxic dermatitis $(n=1)$, postinflammatory pigment alteration $(\mathrm{n}=1)$, interstitial granulomatous dermatitis $(\mathrm{n}=1)$, Staphylococcus folliculitis $(\mathrm{n}=1)$ and morphea $(\mathrm{n}=1)$.

Performance of flow cytometry analyses of skin biopsy samples. The sensitivity and specificity of each diagnostic modality were calculated for patients on the basis of lesion type and were divided into 4 groups on the basis of clinical presentation (Table II). The per patient sensitivity of flow cytometry analyses of skin biopsy specimens was 55\% for clinical patches, $75 \%$ for plaques, and $0 \%$ for nodular disease, and $100 \%$ for erythroderma, for an overall sensitivity of $66 \%$. The per patient specificity of flow cytometry analyses was $100 \%$ for patches, plaques, nodular disease, and erythroderma, with an overall specificity of $100 \%$. Specimens with insufficient cellularity were excluded from the final calculations; these included $33 \%$ of patches, $9 \%$ of plaques, $0 \%$ of nodules/tumors, and $33 \%$ of erythrodermic skin. Therefore, a total of $21 \%$ of specimens submitted for flow cytometry analysis were insufficient.

Performance of molecular analyses of skin biopsy samples. A total of $72 \%$ of skin biopsies had sufficient DNA for performance of TCR-PCR. The sensitivity of TCR-PCR analyses of skin biopsy samples was 55\% for patients with clinical patches, $82 \%$ for plaques, and $0 \%$ for both the 1 tumor-stage CTCL case and the 1 erythrodermic CTCL case; overall sensitivity was $61 \%$. The specificity of TCR-PCR analyses was $66 \%$ for patients with patches, $50 \%$ for plaques, $100 \%$ for nodular disease, and $50 \%$ for erythroderma, for an overall specificity of $64 \%$. TCR-HTS on skin biopsy samples had a per patient sensitivity of $25 \%$ for clinical patches, $89 \%$ for plaques, and 
Table II. Sensitivity and specificity of flow cytometry, TCR-PCR and TCR-HTS analyses of skin biopsy specimens.

\begin{tabular}{|c|c|c|c|c|c|}
\hline $\begin{array}{l}\text { Clinical } \\
\text { morphology }\end{array}$ & Study & $\begin{array}{c}\text { No. of adequate } \\
\text { specimens/patients } \\
\text { tested (\%) }\end{array}$ & $\begin{array}{c}\text { No. of positive } \\
\text { specimens/adequate } \\
\text { specimens } \%\end{array}$ & $\begin{array}{c}\text { Per patient sensitivity } \\
\text { in adequate } \\
\text { specimens, } \%\end{array}$ & $\begin{array}{c}\text { Per patient } \\
\text { specificity } \\
\text { in adequate } \\
\text { specimens, \% }\end{array}$ \\
\hline \multirow[t]{3}{*}{ Patch } & Flow cytometry & $14 / 21 \quad(67)$ & $5 / 14(36)$ & 55 & 100 \\
\hline & TCR-PCR & $15 / 20 \quad(75)$ & $7 / 15(47)$ & 55 & 66 \\
\hline & TCR-HTS & $7 / 7 \quad(100)$ & $1 / 7 \quad(14)$ & 25 & 100 \\
\hline \multirow[t]{3}{*}{ Plaque } & Flow cytometry & $21 / 23$ & $9 / 21(43)$ & 75 & 100 \\
\hline & TCR-PCR & $19 / 20$ & $15 / 19(79)$ & 82 & 50 \\
\hline & TCR-HTS & $17 / 14(100)$ & $9 / 14(64)$ & 89 & 80 \\
\hline \multirow[t]{3}{*}{ Nodule/tumor } & Flow cytometry & $3 / 3 \quad(100)$ & $0 / 3 \quad(0)$ & 0 & 100 \\
\hline & TCR-PCR & $3 / 3 \quad(100)$ & $0 / 3 \quad(0)$ & 0 & 100 \\
\hline & TCR-HTS & $1 / 1 \quad(100)$ & $0 / 1 \quad(0)$ & 0 & NP \\
\hline \multirow[t]{3}{*}{ Erythroderma } & Flow cytometry & $4 / 6 \quad(67)$ & $2 / 4 \quad(50)$ & 100 & 100 \\
\hline & TCR-PCR & $4 / 3 \quad(100)$ & $1 / 3 \quad(33)$ & 0 & 50 \\
\hline & TCR-HTS & $1 / 1 \quad(100)$ & $0 / 1 \quad(0)$ & 0 & $\mathrm{NP}$ \\
\hline \multirow[t]{3}{*}{ All lesions } & Flow cytometry & $42 / 53(100)$ & $16 / 42(38)$ & 66 & 100 \\
\hline & TCR-PCR & $41 / 46 \quad(89)$ & $23 / 41(56)$ & 61 & 64 \\
\hline & TCR-HTS & $26 / 23(100)$ & $10 / 23(43)$ & 60 & 87 \\
\hline
\end{tabular}

NP, not performed; TCR-HTS, T-cell receptor gene high-throughput sequencing; TCR-PCR, T-cell receptor gene rearrangement by polymerase chain reaction.

$0 \%$ for the 1 erythrodermic CTCL case tested, for an overall sensitivity of $60 \%$. TCR-HTS analyses had a specificity of $100 \%$ for patients with patches and $80 \%$ for plaques, with an overall specificity of $87 \%$. Samples submitted for TCR had a higher rate of sufficient DNA: $89 \%$ for PCR and 100\% for HTS, although inadequate specimens were more frequent with patch-stage disease $(25 \%)$. There were 16 patients with concurrent PCR and HTS results; these were concordant in $75 \%$ of cases. In the four discordant cases, there were equal numbers of false-positive and false-negative results for both types of testing.

Performance of flow cytometry analyses of peripheral blood samples. Table III demonstrates the yield, sensitivity and specificity of peripheral blood analysis by flow cytometry and molecular analysis in the lesion subgroups and entire group of patients. Flow cytometry analyses of peripheral blood samples identified an abnormal clone in $23 \%$ of patients diagnosed with patch-stage CTCL, $0 \%$ with plaque-stage CTCL, $0 \%$ with tumor-stage CTCL, and $50 \%$ with erythrodermic CTCL/Sèzary syndrome, for an overall sensitivity of $14 \%$. The specificity of flow cytometry analyses of peripheral blood samples was $85 \%$ for clinical patches, $83 \%$ for plaques, $100 \%$ for nodular disease, and $75 \%$ for erythroderma, for an overall specificity of $88 \%$.

Performance of molecular analyses of peripheral blood samples. TCR-PCR analyses of peripheral blood samples were positive in $38 \%$ of patients diagnosed with patch-stage CTCL, 36\% with plaque-stage disease, $0 \%$ with tumor-stage disease, and $75 \%$ diagnosed with erythrodermic CTCL/Sèzary syndrome, for an overall sensitivity of $40 \%$. The specificity of TCR-PCR analyses of peripheral blood samples was $71 \%$ for clinical patches, $57 \%$ for plaques, $0 \%$ for nodular disease, and $100 \%$ for erythroderma, for an overall specificity of $61 \%$.

TCR-HTS analyses of peripheral blood samples was positive in $0 \%$ of patients diagnosed with patch-stage CTCL and $50 \%$ with plaque-stage CTCL; TCR-HTS analyses were not performed in cases of tumor-stage CTCL or erythrodermic CTCL/Sèzary syndrome. The overall sensitivity of TCR-HTS analyses was $30 \%$. The specificity of TCR-HTS analyses of peripheral blood samples was $100 \%$ for patches, $100 \%$ for plaques, and $100 \%$ for nodular disease. TCR-HTS analyses were not performed on peripheral blood samples of patients with erythrodermic skin (Table III). Forty-nine (49) patients had concurrent peripheral blood flow cytometry and clonality analysis, either by PCR or HTS. Thirty-four (34) (69\%) of these patients had concordant results. The most common cause of discordant results was false-negative flow cytometry (9/15 discordant results) followed by false-positive molecular results (4/15 discordant samples) and false-negative molecular results (2/15 samples).

\section{Discussion}

Cutaneous T-cell lymphoma (CTCL) is a cutaneous lymphoma that clinically mimics a variety of inflammatory dermatoses. A skin biopsy demonstrating characteristic histologic features, including a predominant $\mathrm{CD} 4^{+}$superficial lymphoid infiltrate with atypical cerebriform nuclei, epidermotropism, Pautrier microabscesses, and loss of $\geq 1$ pan-T-cell antigens (CD2, CD3, CD5), can be used to affirm the diagnosis (8). Although these 
Table III. Sensitivity and specificity of flow cytometry, TCR-PCR, and TCR-HTS analyses of peripheral blood.

\begin{tabular}{llccc}
\hline Clinical morphology & \multicolumn{1}{c}{ Study } & $\begin{array}{c}\text { No. of positive } \\
\text { specimens/total } \\
\text { specimens }(\%)\end{array}$ & $\begin{array}{c}\text { Per patient } \\
\text { sensitivity in } \\
\text { adequate specimens, } \%\end{array}$ & $\begin{array}{c}\text { Per patient } \\
\text { specificity in } \\
\text { adequate specimens, } \%\end{array}$ \\
\hline Patch & Flow cytometry & $4 / 20(20)$ & 23 & 85 \\
& TCR-PCR & $7 / 20(35)$ & 38 & 71 \\
Plaque & TCR-HTS & $0 / 5(0)$ & 0 & 100 \\
& Flow cytometry & $0 / 22(0)$ & 0 & 83 \\
Nodule/tumor & TCR-PCR & $8 / 21(38)$ & 36 & 57 \\
& TCR-HTS & $3 / 9(33)$ & 50 & 100 \\
Erythroderma & Flow cytometry & $0 / 3(0)$ & 0 & 0 \\
& TCR-PCR & $2 / 3(66)$ & 0 & 0 \\
All lesions & TCR-HTS & $0 / 2(0)$ & 50 & 75 \\
& Flow cytometry & $3 / 6(50)$ & NP & 100 \\
& TCR-PCR & $3 / 6(50)$ & 14 & NP \\
& TCR-HTS & NP & 40 & 61 \\
& Flow cytometry & $7 / 53(13)$ & 30 & 100 \\
\hline
\end{tabular}

NP, not performed; TCR-HTS, T-cell receptor gene high-throughput sequencing; TCR-PCR, T-cell receptor gene rearrangement by polymerase chain reaction.

pathognomonic features are seen in well-established cases of CTCL, they are rarely observed in cases of early-stage CTCL, in which differentiation between neoplastic and reactive T-cell infiltrates is difficult.

The diagnostic workup for suspected CTCL includes the use of ancillary diagnostic techniques, which have been shown to have promising results in the diagnosis of CTCL $(26,27)$. The identification of T-cell monoclonality within the lymphoid infiltrate can provide strong evidence of the diagnosis of CTCL. In well-established CTCL cases, T-cell receptor (TCR) has been shown to detect T-cell monoclonality in 52 to $90 \%$ of established CTCL biopsy specimens (5,28-30). Kirsch et al demonstrated superior results using high-throughput sequencing (HTS), which detected expanded T-cell clones in $100 \%$ (46 out of 46) of established CTCL cases (19).

Little is known about the usefulness of these ancillary studies in early or pathologically borderline/equivocal CTCL cases. Ashton-Key et al analyzed clinically suspicious, histologically borderline CTCL biopsy specimens of lesions from 22 patients who subsequently developed mycosis fungoides and 32 newly suspected CTCL patients. TCR detected monoclonality in 50\% (11/22) and 19\% (6/32) of cases, respectively (13). The use of HTS in examining clinically suspicious, histologically borderline lesions has not been studied.

Once a diagnosis of CTCL is established, additional diagnostic testing is conducted in accordance with current National Comprehensive Cancer Network guidelines for the workup and staging of cutaneous lymphomas (31). These diagnostic tests include PET/CT scans and laboratory studies, such as complete blood count, differential, comprehensive metabolic panel, lactic dehydrogenase, serum protein electrophoresis/quantitative immunoglobulins, molecular testing with cytogenetics, fluorescence in situ hybridization, flow cytometry, and viral studies (32), as clinically indicated. A bone marrow biopsy is also performed in select cases of CTCL (33).

The majority of patients in our cohort presented with low-volume patch or plaque-stage disease. For tissue evaluation, the sensitivity of each technique was similar for the population as a whole, while flow cytometry was most specific. Although flow cytometry was useful for analyzing skin biopsy specimens from patients with plaques and erythroderma, it suffered from low sensitivity for patch-stage disease and had a high rate of insufficient tissue $(33 \%)$. We no longer perform flow cytometry of patch-stage skin lesions. TCR-PCR remains a valuable technique to assess the tissue of patients with suspected CTCL, especially with patch and plaque lesions, but had a lower sensitivity in patch-stage disease and a high rate of insufficient tissue (25\%). Since our population was skewed towards patients with suspected malignancy, we do not have adequate numbers of benign diagnoses to make valid conclusions about the specificity of TCR-PCR. Compared to TCR-PCR, TCR-HTS offered similar sensitivity but had greater specificity in patients with plaque but not patch lesions. Both TCR-PCR and TCR-HTS had greater sensitivity than flow cytometry in assessing patients with raised lesions; flow cytometry offered greater specificity than TCR-PCR among those with clinically raised lesions. The PCR and HTS techniques showed a high rate of concordance $(75 \%)$ in the low number of samples concurrently tested. For all of these tests, it must be emphasized that our reported performance characteristics can only be generalized to patients with early or partially treated disease, as this population was the focus of this study. Furthermore, the low numbers of patients tested with TCR-HTS limits 
drawing strong conclusions about test performance, although the results are promising.

With the exception of erythrodermic patients, the overall rate of positive TCR and flow cytometry studies performed on peripheral blood specimens was low. These data suggest that flow cytometry analyses of peripheral blood may safely be eliminated from the workup of patients with patch and plaque disease; the low numbers of patients with nodules/tumor disease precluded the evaluation of this technique. In 9 cases, TCR-PCR analyses performed on blood yielded a positive result when concurrent flow cytometry was negative. Although TCR-PCR was more sensitive than TCR-HTS, TCR-HTS had a significantly higher specificity when performed on peripheral blood. For patients with positive TCR-PCR but negative flow cytometry of peripheral blood, a comparison of a TCR peak(s) with the tissue specimen is suggested to evaluate for matching peaks. Indeed, all 3 positive TCR-HTS results had matching peaks in tissue and peripheral blood; in all 3 of these flow cytometry was negative. An accurate comparison of TCR-PCR peaks may often be challenging, whereas TCR-HTS identifies matching gene sequences with superior specificity and has the ability to reveal low levels of dominant sequences found in skin biopsies as well as within corresponding peripheral blood specimens.

Given the rising health care costs in the US, a cost-conscious approach must be considered in diagnostic medicine. Despite the decrease in the cost of next-generation sequencing in the last decade, the techniques of established flow cytometry methods and PCR-based studies are less costly. However, the higher rates of insufficient tissue associated with flow cytometry and PCR studies, leading to the need for additional biopsies, may negate any cost difference.

These data suggest that molecular analyses remain a useful tool for evaluating patients with suspected CTCL. In the 16 patients tested, TCR by HTS showed better specificity for both the skin biopsy and peripheral blood samples than the traditional PCR method, although sensitivities were similar. By identifying the gene sequences present in lesional tissue, HTS may be superior for monitoring for recurrence and minimal residual disease. Flow cytometry of tissue can be a useful adjunct to molecular analyses for patients with plaque, tumor, or erythrodermic lesions. In our limited sample, flow cytometry analyses of peripheral blood samples showed relatively low sensitivity and moderate specificity in patch, plaque, and tumor disease, and flow cytometry analyses of peripheral blood remains a useful tool for evaluating patients with erythroderma. Although our findings are limited by the low number of cases studied, they point to the continued need for more sensitive tests for early-stage CTCL.

\section{Acknowledgements}

We thank Paul Fletcher and Daley Drucker (Moffitt Cancer Center) for editorial assistance. They were not compensated beyond their regular salaries.

\section{Funding}

No funding was received.

\section{Availability of data and materials}

All data generated and/or analyzed during the current study are included in this published article. The high-throughput sequencing that was performed on a subset of patients was obtained through testing at a commercial laboratory, and results are included in the article.

\section{Authors' contributions}

JLM carried out the creation of the research project, data gathering and analysis, substantial preparation and final approval of manuscript and is the corresponding author. JDG carried out the data gathering and analysis, preparation of manuscript, and gave final approval of the manuscript. SM conducted the data gathering and analysis, preparation of manuscript, and gave final approval of the manuscript. AK carried out the data collection and analysis, and gave final approval of the manuscript. LS and LSV achieved conception of the study and gave final approval of the manuscript. ES, HZ and XZ made contribution to the methods, analysis of data, and gave final approval of the manuscript. PLC conducted analysis of the data, and gave final approval of the manuscript. All authors read and approved the manuscript and agree to be accountable for all aspects of the research in ensuring that the accuracy or integrity of any part of the work are appropriately investigated and resolved.

\section{Ethics approval and consent to participate}

This study was carried out with the approval of the institutional ethics review boards: Moffitt Cancer Center Scientific Review Committee (MCC 19315) and University of South Florida Bulls IRB (Pro00031911).

\section{Patient consent for publication}

According to institutional guidelines, the patient images contained herein show no personally identifying data and therefore the requirement for patient consent was waived.

\section{Competing interests}

The authors declare that the research was conducted in the absence of any commercial or financial relationships that could be construed as a potential conflict of interest.

\section{References}

1. Swerdlow SH, Campo E, Harris NL, Jaffe ES, Pileri SA, Stein H and Thiele J: World Health Organization Classification of Tumours of Hematopoietic and Lymphoid Tissue., IARC Press, Lyon, 2008.

2. Cerroni L: Lymphoproliferative lesions of the skin. J Clin Pathol 59: 813-826, 2006.

3. Willemze R and Meijer CJ: Classification of cutaneous T-cell lymphoma: From Alibert to WHO-EORTC. J Cutan Pathol 33 (Suppl 1): 18-26, 2006.

4. Olsen EA: Evaluation, diagnosis, and staging of cutaneous lymphoma. Dermatol Clin 33: 643-654, 2015.

5. Algara P, Soria C, Martinez P, Sanchez L, Villuendas R, Garcia P, Lopez C, Orradre JL and Piris MA: Value of PCR detection of TCR gamma gene rearrangement in the diagnosis of cutaneous lymphocytic infiltrates. Diagn Mol Pathol 3: 275-282, 1994. 
6. Galper SL, Smith BD and Wilson LD: Wilson, Diagnosis and management of mycosis fungoides. Oncology (Williston Park) 24: 491-501, 2010.

7. Willemze R, Jaffe ES, Burg G, Cerroni L, Berti E, Swerdlow SH, Ralfkiaer E, Chimenti S, Diaz-Perez JL, Duncan LM, et al: WHO-EORTC classification for cutaneous lymphomas. Blood 105: 3768-3785, 2005

8. Cerroni L: Mycosis fungoides-clinical and histopathologic features, differential diagnosis, and treatment. Semin Cutan Med Surg 37: 2-10, 2018.

9. Jawed SI, Myskowski PL, Horwitz S, Moskowitz A and Querfeld C: Primary cutaneous T-cell lymphoma (mycosis fungoides and Sézary syndrome): Part I. Diagnosis: Clinical and histopathologic features and new molecular and biologic markers. J Am Acad Dermatol 70: 205.e1-16; quiz 221-222, 2014

10. Kempf W and Mitteldorf C: Mitteldorf, pathologic diagnosis of cutaneous lymphomas. Dermatol Clin 33: 655-681, 2015.

11. Hodak E and Amitay-Laish I: Amitay-Laish, Mycosis fungoides: A great imitator. Clin Dermatol 37: 255-267, 2019.

12. Campbell SM, Peters SB, Zirwas MJ and Wong HK: Immunophenotypic diagnosis of primary cutaneous lymphomas: A review for the practicing dermatologist. J Clin Aesthet Dermatol 3: 21-25, 2010.

13. Ashton-Key M, Diss TC, Du MQ, Kirkham N, Wotherspoon A and Isaacson PG: The value of the polymerase chain reaction in the diagnosis of cutaneous T-cell infiltrates. Am J Surg Pathol 21: 743-747, 1997.

14. Alaibac M, Pigozzi B, Belloni-Fortina A, Michelotto A Saponeri A and Peserico A: CD7 expression in reactive and malignant human skin T-lymphocytes. Anticancer Res 23 2707-2710, 2003

15. Moll M, Reinhold U, Kukel S, Abken H, Müller R, Oltermann I and Kreysel HW: CD7-negative helper T cells accumulate in inflammatory skin lesions. J Invest Dermatol 102: 328-332, 1994

16. Lu C, Zhang J, Nagahawatte P, Easton J, Lee S, Liu Z, Ding L, Wyczalkowski MA, Valentine M, Navid F, et al: The genomic landscape of childhood and adolescent melanoma. J Invest Dermatol 135: 816-823, 2015

17. Skov AG and Gniadecki R: Gniadecki, Delay in the histopathologic diagnosis of mycosis fungoides. Acta Derm Venereol 95 472-475, 2015

18. van Doorn R, Van Haselen CW, van Voorst Vader PC, Geerts ML, Heule F, de Rie M, Steijlen PM, Dekker SK, van Vloten WA and Willemze R: Mycosis fungoides: Disease evolution and prognosis of 309 Dutch patients. Arch Dermatol 136: 504-510, 2000.

19. Kirsch IR, Watanabe R, O'Malley JT, Williamson DW, Scott LL, Elco CP, Teague JE, Gehad A, Lowry EL, LeBoeuf NR, et al: TCR sequencing facilitates diagnosis and identifies mature T cells as the cell of origin in CTCL. Sci Transl Med 7: 308ra158, 2015.

20. Maitre E, Le-Page AL, Comoz F, Truquet F, Damaj G, Cornet E, Verneuil L, Salaün V and Troussard X: Usefulness of flow cytometry for the detection of cutaneous localization in malignan hematologic disorders. Cytometry B Clin Cytom 96: 283-293. 2019.
21. Bawazir MA, Almohideb M, Walsh S, Shear NH and Alhusayen R: Early-stage mycosis fungoides screening investigations: A retrospective analysis of 440 cases. J Eur Acad Dermatol Venereol 32: e217-e218, 2018.

22. Horna P, Kurant D, Sokol L, Sotomayor EM, Moscinski L and Glass LF: Flow cytometric identification of immunophenotypically aberrant T-cell clusters on skin shave biopsy specimens from patients with mycosis fungoides. Am J Clin Pathol 143 785-796, 2015.

23. Rea B, Haun P, Emerson R, Vignali M, Farooqi M, Samimi S, Elenitsas R, Kirsch I and Bagg A: Role of high-throughput sequencing in the diagnosis of cutaneous T-cell lymphoma. J Clin Pathol 71: 814-820, 2018.

24. de Masson A, O'Malley JT, Elco CP, Garcia SS, Divito SJ, Lowry EL, Tawa M, Fisher DC, Devlin PM and Teague JE: High-throughput sequencing of the T cell receptor $\beta$ gene identifies aggressive early-stage mycosis fungoides. Sci Transl Med 10: eaar5894, 2018.

25. Weng WK, Armstrong R, Arai S, Desmarais C, Hoppe R and Kim YH: Minimal residual disease monitoring with high-throughput sequencing of $\mathrm{T}$ cell receptors in cutaneous T cell lymphoma. Sci Transl Med 5: 214ra171, 2013.

26. Torres-Cabala CA: Diagnosis of T-cell lymphoid proliferations of the skin: Putting all the pieces together. Mod Pathol 33: (Suppl 1): 83-95, 2019.

27. Cocks M, Porcu P, Wick MR and Gru AA: Recent advances in cutaneous T-cell lymphoma: Diagnostic and prognostic considerations. Surg Pathol Clin 12: 783-803, 2019.

28. Liebmann RD, Anderson B, McCarthy KP and Chow JW: The polymerase chain reaction in the diagnosis of early mycosis fungoides. J Pathol 182: 282-287, 1997.

29. Theodorou I, Delfau-Larue MH, Bigorgne C, Lahet C, Cochet G, Bagot M, Wechsler J and Farcet JP: Cutaneous T-cell infiltrates: Analysis of T-cell receptor gamma gene rearrangement by polymerase chain reaction and denaturing gradient gel electrophoresis. Blood 86: 305-310, 1995.

30. Wood GS, Tung RM, Haeffner AC, Crooks CF, Liao S, Orozco R, Veelken H, Kadin ME, Koh H, Heald P, et al: Detection of clonal T-cell receptor gamma gene rearrangements in early mycosis fungoides/Sezary syndrome by polymerase chain reaction and denaturing gradient gel electrophoresis (PCR/DGGE). J Invest Dermatol 103: 34-41, 1994.

31. Zelenetz AD: Guidelines for NHL: Updates to the management of diffuse large B-cell lymphoma and new guidelines for primary cutaneous $\mathrm{CD} 30^{+} \mathrm{T}$-cell lymphoproliferative disorders and T-cell large granular lymphocytic leukemia. J Natl Compr Canc Netw 12 (Suppl 5): 797-800, 2014.

32. Liu WP, Song YQ, Zheng W, Wang XP, Ding N and Zhu J: Aggressive behavior and elevated lactate dehydrogenase at baseline confer inferior prognosis in patients with primary cutaneous lymphoma. Clin Lymphoma Myeloma Leuk 13: 534-540, 2013.

33. Quereux G, Frot AS, Brocard A, Leux C, Renaut JJ and Dreno B: Routine bone marrow biopsy in the initial evaluation of primary cutaneous B-cell lymphoma does not appear justified. Eur J Dermatol 19: 216-220, 2009. 